\title{
MÁS ALLÁ DEL CONCEPTO: EXPERIENCIAS Y REFLEXIONES EN TORNO A LA PARTICIPACIÓN JUVENIL ESTUDIANTIL
}

\author{
SEBAstiÁn EscobAR GONZÁLEZ*1 \\ HERMAN PEZO HOCES**
}

\begin{abstract}
RESUMEN
La participación se constituye como una noción polémica y polisémica, pues posee un abanico amplio de interpretaciones. Ante esta trampa conceptual, en el siguiente artículo profundizamos en el ejercicio de la participación juvenil estudiantil, buscando desenmarañar su entendimiento. Recurrimos a materiales cualitativos en los que participaron jóvenes estudiantes secundarios/as y docentes de enseñanza media de liceos municipales, utilizando el análisis de contenido temático. Los principales hallazgos dan cuenta de que existen diversas prácticas que permiten avanzar hacia una participación auténtica y sustantiva, reconociendo, a su vez, ejercicios que se configuran en su antítesis, a pesar de ser consideradas como tal.
\end{abstract}

\footnotetext{
PALABRAS CLAVE: JUVENTUDES, INSTITUCIÓN ESCOLAR, PARTICIPACIÓN, ADULTOCENTRISMO, CULTURAS JUVENILES, CULTURA ESCOLAR
}

\footnotetext{
* Licenciado en Educación, Pontificia Universidad Católica de Chile, y Magíster en Ciencias Sociales, Universidad de Chile. Profesor de la Facultad de Educación de la Universidad Finis Terrae y miembro del Núcleo de Investigación y Acción en Juventudes, Universidad de Chile. Correo electrónico: siescobar@uc.cl

**Trabajador social, Universidad Alberto Hurtado, Chile. Investigador Núcleo de Investigación y Acción en Juventudes, Universidad de Chile. Correo electrónico: hermanfabian.pezo@gmail.com
} 


\title{
ALÉM DO CONCEITO: EXPERIÊNCIAS E REFLEXÕES SOBRE A PARTICIPAÇÃO JUVENIL ESTUDANTIL
}

\begin{abstract}
RESUMO
A participação é constituída como uma noção polêmica e polissêmica, uma vez que possui uma ampla gama de interpretações. Por causa desta armadilha conceitual, no seguinte artigo, aprofundamos no exercício da participação juvenil estudantil, com o objetivo de desvendar sua compreensão. Recorreu-se a materiais qualitativos onde participaram jovens estudantes e professores das escolas municipais de ensino médio, utilizando a análise de conteúdo temática. Os principais achados mostram que existem várias práticas que permitem avançar para uma participação autêntica e substantiva, reconhecendo, por sua vez, exercícios configurados em sua antítese, apesar de serem considerados dessa maneira.
\end{abstract}

PALAVRAS-CHAVE: JUVENTUDES, INSTITUIÇÃO ESCOLAR, PARTICIPAÇÃO, ADULTOCENTRISMO, CULTURAS JUVENIS, CULTURA ESCOLAR

\section{BEYOND THE CONCEPT: EXPERIENCES AND REFLECTIONS ON YOUTH STUDENT PARTICIPATION}

\begin{abstract}
Participation is established as a polysemic and controversial notion, as it entails a wide spectrum of interpretations. In the face of this conceptual trap, the following article delves into the exercise of youth student participation, in order to reveal its understanding. We resorted to qualitative materials (content analysis) collected from young secondary students and teachers from state schools. The main findings present the existence of diverse practices paving the path to an authentic and substantial participation that recognizes, in parallel, those practices that are still considered as such in spite of being antithetical.
\end{abstract}

KEYWORDS: YOUTHS, SCHOOL, PARTICIPATION, ADULTCENTRISM, YOUTH CULTURES, SCHOOL CULTURE 


\section{INTRODUCCIÓN}

Como acercamiento inicial, la concepción etimológica de participación proviene del latín tardío participatio, que indica la acción y afecto de tomar parte en algo, o hacer parte a alguien más sobre algo (Corominas, 1954). Pese a esta referencia, es posible aseverar que no existe una configuración homogénea sobre su comprensión y abordaje, constituyéndose más bien como un concepto polisémico y polémico, el que se encuentra en permanente disputa y debate público al ser una noción altamente deseable en sociedades que se asumen democráticas.

En ese sentido, dependiendo del enfoque que se adopte, se considerarán determinados aspectos que entran en juego a la hora de hablar de participación. En relación con ello, algunas perspectivas pondrán énfasis en torno a los niveles o escalas: informativa, consultiva, propositiva, resolutiva, ejecutiva y evaluativa (Hart, 1993, Krauskopf, 2003); en sus variantes o tipos, según el campo donde se despliega: participación social, ciudadana, política, electoral, etc. (Palenzuela, 2016; Bañez, 1999); en las condiciones contextuales y relacionales que facilitan u obstaculizan el ejercicio participativo (Palma, 1998; Pezo, 2019); en su sentido político y transformador (Freire, 1993) o bien aquellas que se focalizan en la participación de actores e instancias específicas (Núcleo Juventudes, 2016).

Frente a esta pluralidad epistémica y teórica-metodológica de la participación, algunos/as autores/as han alertado el carácter jabonoso que tendría, planteando que se trataría de una noción difícil de asir. Así, las denominaciones de significado flotante (Anderson, 2001) o contenido vacío de la participación (Franco, B., Franco, P. y Guilló, 2007) ponen de relieve la multiplicidad de interpretaciones y ambigüedades que genera, requiriendo un esfuerzo analítico para precisar qué se entiende por ella. Ante esto, asumimos que la participación solo tiene cabida en función de su despliegue, por tal razón, lejos de considerarla como un ejercicio abstracto, se trata de reconocer las condiciones contextuales y relaciones de poder donde se ejerce, y simultáneamente, considerar la capacidad con la que cuentan los sujetos para involucrarse voluntariamente e incidir en el entramado social del que forman parte.

En consideración a ello, en las últimas décadas, la participación juvenil ha cobrado interés y preocupación en el mundo social, académico y político, siendo abordada y promovida con diversas visiones y fines. De esta manera, han emergido interpretaciones que tienden a construir imágenes contrapuestas respecto a la participación de la población juvenil. En esa línea, es posible señalar que existen claves interpretativas que tienden a plantear una supuesta desafección o apatía que afectaría a las y los jóvenes frente a otras lecturas, que insisten en la necesidad de ampliar la mirada reconociendo la diversidad de 
intereses y espacios donde las juventudes se hacen parte activamente (Escobar, 2019; Palenzuela, 2016, 2019; Zarzuri, 2016).

De esta forma, se trata de reconocer que, si bien en Chile los resultados de diversos estudios dan cuenta de una baja participación electoral o política institucional en las y los jóvenes (Comisión Formación Ciudadana, 2005; INJUV, 2014), en el mismo movimiento, los resultados del Instituto Nacional de Juventud (INJUV, 2017) posibilitan considerar la participación juvenil desde otra óptica, donde se logra constatar una participación persistente en espacios comunitarios, sociales y organizativos que van más allá de lo electoral.

A su vez, es posible considerar que, en el último período, las juventudes se han constituido como un actor que participa y cuestiona la congruencia de las diversas instituciones sociales, construyendo demandas que buscan mejorar las problemáticas que les atañen. Así, en el contexto chileno, ejemplo de ello son las movilizaciones estudiantiles de los años 2006-2011, movimiento feminista 2018 y el reciente estallido social de octubre de 2019. Por tanto, la participación juvenil tiende a desplegarse en aquellos procesos que les involucran, y donde perciben que su palabra es considerada como relevante (Duarte y Álvarez, 2016). Así entonces, supone reconocer que la participación no solo se agota en la política tradicional, sino que discurre en los diversos planos de lo político (Hernández, 2019).

Respecto al sistema educativo, la participación juvenil tampoco ha estado exenta de tensiones y desafíos. En ese sentido, diversas investigaciones han dado cuenta de una serie de desencuentros y conflictos entre la cultura juvenil y la cultura escolar, principalmente porque la institución escolar tendría a cerrarse y producir una tendencia homogeneizadora respecto de las experiencias que portan las juventudes, más allá del régimen de enseñanza y disciplinar de la institución escolar (Dubet y Martuccelli, 1998; Molina y Sandoval, 2006; Tenti, 2000).

Lo anterior estaría reforzado por las formas en que se configuran y establecen las relaciones intergeneracionales en el liceo, donde se constatan puentes rotos en las relaciones entre adultos y jóvenes (Duarte, 2001) mediadas por una serie de máscaras y corazas que dificultan posibilidades de acercamiento y vinculación profundas. De esta manera, las lógicas adultocéntricas en los modos de relación generacional articulados a los procesos de disciplinamiento que caracterizan al dispositivo educativo condicionan el despliegue de la participación de las y los jóvenes estudiantes (Dussel, 2005; Pezo, 2019).

Frente a este escenario, el siguiente artículo emerge a partir de dos procesos de investigación con metodología cualitativa llevados a cabo durante el año 2017, los que tenían por objeto de estudio abordar la participación juvenil en el contexto escolar. Así, una primera investigación centrada en la cultura escolar buscaba analizar las condiciones que facilitaban-obstaculizaban 
su ejercicio. La segunda, enfocada en la cultura juvenil, ahondaba directamente en los procesos de participación que jóvenes desarrollaban fuera del espacio escolar y cómo estas formas se vinculaban con la cultura escolar en la que estaban inmersos. De esta manera, la selección de ambas experiencias se realizó bajo un muestreo intencional (Andreu, 2001), buscando profundizar en la noción de participación juvenil estudiantil desde los discursos de jóvenes y adultos/as en el contexto escolar. Para ello, recurrimos a los discursos de actores educativos, grupos focales y entrevistas a estudiantes y docentes de liceos municipales, cincuenta y seis discursos juveniles y siete voces adultas ${ }^{2}$. El análisis de contenido temático (Andreu, 2001) posibilitó interpretar el texto a partir de su contenido latente y sentido oculto, para «buscar unidades en que aparece una determinada temática» (p. 20).

A continuación, presentamos los principales hallazgos de este artículo, exponiendo dos tramas analíticas de la participación. Así, un primer eje tensiona las modalidades con las que es capturada la participación juvenil estudiantil en las instituciones educativas, las que se constituyen más bien como artificios de participación. En un segundo lente analítico, ahondamos en algunas pistas para avanzar hacia lo que hemos denominado como participación auténtica y sustantiva, poniendo énfasis en aquellas prácticas de participación que emergen de manera genuina. Finalmente, cerramos con un conjunto de reflexiones que invitan a repensar la participación en el liceo, dando fuerza al desarrollo de diálogos intergeneracionales para subvertir el orden asimétrico de las relaciones sociales que allí operan.

\section{SIMULACROS SIMBÓLICOS DE PARTICIPACIÓN}

La noción de simulacro simbólico de participación (Palma, 1998) pone en discusión aquellas experiencias que, a pesar de ser promovidas formalmente en los espacios educativos, no logran traducirse necesariamente como un ejercicio genuino y fecundo, pues carecen de una injerencia en la toma de decisiones por parte de los actores juveniles, lo que ha sido considerado como una falsa participación que se ejerce en los espacios escolares (Jara, 2011). En ese sentido, en las instituciones educacionales, la participación a menudo es capturada bajo formas no democráticas o manipulativas, aunque estas no necesariamente responden a una intencionalidad negativa (Anderson, 2001). Bajo este contexto, presentamos algunas variantes que son posibles de

\footnotetext{
${ }^{2}$ Se realizaron diez grupos focales y cinco entrevistas a jóvenes estudiantes de tercer y cuarto año medio. Además, se desarrollaron siete entrevistas a docentes. Los sujetos participantes provenían de liceos municipales de la Región Metropolitana de Chile. Para efectos de este artículo, hemos identificado a cada hablante según la comuna del liceo, resguardando la confiabilidad de la información, tal como fue expresado en los consentimientos informados.
} 
reconocer desde este pliegue analítico, ofreciendo tipologías que intentan develar aquellas prácticas que se configuran como antítesis de la participación de mundos juveniles.

\subsection{PARTICIPACión COSIFICADA}

Cuando se plantea la noción de participación desde las voces adultas, una tendencia que emerge es que su forma de comprensión y representación suele delimitarse como un elemento que aparece en ciertos espacios y momentos. Así, la participación juvenil estudiantil es dibujada en determinadas instancias o actividades reconocidas desde el ámbito formal-institucional, principalmente vinculada a instancias recreativas, deportivas o al aula de clases. De esta manera, corre el riesgo de quedar enmarcada únicamente dentro de los parámetros legitimados por la institución escolar.

Yo asocio la participación juvenil como agrupaciones de estudiantes en torno a diferentes actividades, por ejemplo, cuando yo venía los días sábado de repente para apoyar un taller que se hacía aquí en el liceo, veía muchas actividades dentro del liceo, veía, así como algunos estaban haciendo vóley, otros estaban haciendo básquetbol, otros a la vez un taller de malabarismo (...) desde ahí había una diversidad de instancias. (Docente adulto varón, Liceo Puente Alto)

Ya, yo entiendo que participación, una participación en clases, participación en las actividades del colegio, no sé po', en lo que se les pueda pedir, como ordenar las salas, decorar sus salas, yo creo que por ese lado hay harta participación. (Docente adulto varón, Liceo de Colina)

Desde este prisma, es posible dar cuenta que la participación no logra ser considerada como un elemento permanente en las dinámicas e interacciones que desarrollan los distintos actores educativos, lo que termina cristalizando su entendimiento a un conjunto limitado de expresiones, provocando que la participación juvenil estudiantil quede construida de manera estática y cosificada.

\subsection{PARTICIPACIÓN INSTRUMENTAL}

Una segunda observación sobre este eje analítico da cuenta de que la participación juvenil estudiantil en el dispositivo educativo es capturada por una racionalidad instrumental (Horkheimer, 2002) que centra su preocupación en torno al cumplimiento de ciertas actividades. En ese sentido, la participación se convierte en un conjunto de instancias protocolares, las que deben ser realizadas para su posterior fiscalización o comprobación. Así, lo que se valora 
desde esta perspectiva es la cantidad de instancias o participantes que logran ser convocadas/os y no necesariamente el acuerdo o la toma de decisiones que se llevan a cabo.

Son básicos [los espacios de participación]... los espacios llegan a ser básicos porque cumplen solamente como si fuera un visto bueno. (Joven varón secundario, Liceo Santiago Centro)

Creo que son súper precarios [los espacios de participación], o sea, hay de todo po'... hay como lugares donde de verdad no tenís ningún espacio de participación, están los colegios como los que yo estuve, que era como si hay centro de alumnos, pero cuando estás en el centro de alumnos te das cuenta de que en verdad no podis hacer ni una hueá y como que lo único que tenís que hacer son las alianzas, y cuando proponís proyecto al final nunca los llevas a cabo porque te tramitan... que los papás, que los profes, que los rectores (...). (Joven mujer secundaria, Liceo de Providencia)

Esta racionalidad instrumental influye negativamente en las percepciones y experiencias de participación juvenil estudiantil, debido a que no solo serían insuficientes o escasas las condiciones para su ejercicio, sino que, además, traerían una pérdida de sentido, pues su preocupación se reduce al cumplimiento burocrático y formal de las actividades, lo que merma una consideración comprehensiva sobre las necesidades, intereses y aportes juveniles.

\subsection{PARTICIPACIÓN AUTORIZADA Y OBLIGATORIA}

La estructura jerárquica de las instituciones educativas pone de manifiesto una tensión inminente sobre la promoción de la participación juvenil estudiantil. En ese sentido, si bien se busca incentivar su ejercicio, generalmente queda consignada a los permisos y limitaciones que establezcan los actores adultos, siendo los equipos directivos y docentes quienes gozan primordialmente de esa potestad. Bajo este contexto, las voces juveniles manifiestan que la participación adopta un carácter obligatorio e impositivo, el que no contempla necesariamente sus intereses, inquietudes y/o deseos.

Entonces, claro, la participación depende mucho de la autorización, por eso te digo, a las actividades, y ya prácticamente, o sea, todo se autoriza, pero tiene que saberse. (Docente adulto varón, Liceo de Santiago Centro)

En nuestro liceo está lo de la burocracia, siempre pasa por personas para hacer algo, ni siquiera puedes hacerlo libre, siempre tenís que mostrar el cronograma, mostrar la actividad que vas a hacer y entonces entregarle todo lo que vas a 
hacer (...) al final te tienden a modificar la organización o te supervisan todo el rato. (Joven mujer secundaria, Liceo de Providencia)

Asimismo, cabe señalar que esta forma de comprender y operacionalizar la participación está sostenida por un modo de relación generacional que se condensa por la matriz de dominio adultocéntrica (Duarte, 2016), la cual establece una asimetría que repercute en una triple dimensión: simbólica, material y corporal-sexual. En ese sentido, la participación debe ser visada por mundos adultos, pues existen ciertos imaginarios sociales que reposan y proyectan un modo particular de participación para las juventudes, lo que justificaría la tutela adulta. Esto conlleva que su ejercicio quede condicionado a ciertos mecanismos de clausura y apertura que operan dentro de la institución escolar por parte de mundos adultos.

Los simulacros de participación juvenil expuestos (cosificada, instrumental y obligatoria), lejos de comprenderlos de manera aislada, también pueden ser reconocidos como modalidades que operan de forma interrelacionada dentro de la realidad educativa. Por tanto, el desafío recae en interrogar críticamente las expresiones que adopta la participación juvenil estudiantil en las instituciones educacionales, reconociendo el poder que cada actor ejerce dentro de ella. Sin embargo, tampoco se trata de adoptar una postura fatalista que renuncie a la posibilidad de ensayar otros modos de comprender y ejercer la participación juvenil estudiantil, por tal motivo, a continuación, presentamos algunas pistas que permiten avanzar hacia una participación auténtica y sustantiva de las juventudes escolarizadas.

\section{Pistas PaRa UNa PaRTICIPACIÓn JUVENIL ESTUdiantiL AUTÉNTICA Y SUSTANTIVA}

Al plantear la participación auténtica (Anderson, 2001), no nos referimos a una participación unívoca, o bien, bajo un enfoque esencialista que vuelva estática su comprensión. Consideramos que se trata de repensarla como un ejercicio auténticamente democrático, lo que desafía considerar algunas preguntas medulares sobre «quién participa, en qué áreas y bajo qué condiciones, y también participación para qué, con qué fin» (Anderson, 2001: 8). Por tanto, supone contemplar que aquellos grupos involucrados en un asunto puedan crear espacios seguros para que todas las voces sean escuchadas. De esta forma, lo que está en juego es el ejercicio de poder que toda relación comporta, por tal motivo, para avanzar hacia una participación sustantiva (Palma, 1998) se requiere necesariamente considerar la influencia en la toma de decisiones y las condiciones para hacerla efectiva. 


\subsection{PARTICIPACIÓN COTIDIANA}

Si bien dentro de la institución escolar existen instancias formales de participación juvenil estudiantil, también es posible reconocerla a partir de múltiples formas que emergen de manera contingente y dinámica dentro del espacio educativo. Esto conlleva a comprender que la participación de las y los jóvenes opera en la acción cotidiana, por ende, se constituye como un proceso continuo que otorga sentido a la experiencia escolar.

También hay sus espacios legales como espacios, como ya están normados dentro de la institución, que vendrían siendo los CODECU, como se llama esto cuando hacemos el consejo de curso, cachái, que ahí se pueden hablar temas y la cuestión, pero no es tan ocupado como en el sentido del día a día, en el día a día igual se puede ver cómo la reflexión va abundando. (Joven varón secundario, Liceo de Santiago Centro)

Porque acá en un recreo de repente uno puede ver a un grupito de personas que está conversando un tema un tanto político, contingente, temas del mismo liceo, entonces, así mismo dándole al duro, se abren los espacios de participación. (Joven varón secundario, Liceo de Santiago Centro)

Comprender la participación como una acción cotidiana, en ningún caso supone desconocer aquellas instancias formales que permiten el involucramiento juvenil, sino que más bien permite robustecer su entendimiento más allá de los mecanismos tradicionales, logrando reconocer prácticas que no suelen ser visualizadas como tal. En ese sentido, complejiza las formas y expresiones que adopta la participación juvenil, y en el mismo movimiento, invita a repensar diversos ámbitos donde las agencias juveniles, desde sus potencialidades, pueden aportar a la construcción de una comunidad educativa auténticamente participativa.

\subsection{PARTICIPACIÓN DIVERSA}

Las y los sujetos juveniles manifiestan que su participación en la institución escolar tiende a ser considerada de forma homogénea $\mathrm{y}$ unidimensional. Esto se encontraría influenciado por la construcción del sujeto joven que deviene desde la institución escolar, la que tiende a anclar la condición de estudiante por sobre otros elementos constitutivos de las realidades juveniles. Lo anterior estaría reforzado también por el elemento etario, donde al existir una similitud con los años de vida entre estudiantes de un cierto nivel educativo, se establecería una especie de homogeneización que invisibilizaría la diversidad inherente entre sujetos. A contracorriente de esta idea, las juventudes dan cuenta de que su participación e intereses se despliegan 
de manera heterogénea, lo que invita a comprender su ejercicio de forma plural y diversa. En ese sentido, sus intereses, trayectorias y modos de habitar en el mundo van variando según la realidad social donde devienen como sujetos.

Como que esperan que seamos todos iguales po', y no po', si al final cada uno es distinto. No porque seamos estudiantes o tengamos la misma edad nos van a gustar las mismas cosas, al final yo siento que nos quieren moldear a todos de la misma forma. (Joven mujer secundaria, Liceo Puente Alto)

Es que acá todos somos distintos, entonces todos participamos en distintas cosas. (Joven mujer secundaria, Liceo Colina)

En consideración a ello, si bien la participación estudiantil pone en el centro de interés a un determinado actor (jóvenes), esto no implica que su comprensión y ejercicio se interpreten desde marcos analíticos totalizadores. En ese sentido, si las juventudes se caracterizan por ser diversas y heterogéneas (Duarte, 2001), resulta importante considerar la participación dentro de esos mismos términos. Esto desafía a los distintos actores educativos a repensar las categorías con las que suele considerarse la participación dentro del espacio educativo.

\subsection{PARTICIPACIÓN TRANSFORMADORA}

Los discursos juveniles dan cuenta de que su ejercicio de participación también está guiado por un componente político, lo que les permite construir propuestas de transformación de las realidades que viven, o de aquellas situaciones que consideran como problemáticas. En ese sentido, se pueden constatar diversas prácticas (individuales y colectivas) que buscan modificar la estructura normativa con la que opera la institución escolar, o iniciativas que intentan integrar elementos que la educación tradicional y neoliberal no contempla.

Entonces eso igual es grato que en este liceo se ve eso, pero no por parte de dirección, no por parte de ningún funcionario, sino que simplemente se da por los cabros que buscan sus propios lugares para reunirse y conversar temas que en el liceo o la educación como tal no da pie a que se cumplan. (Joven varón secundario, Liceo Puente Alto)

Nosotros, cuando mandamos el proyecto educativo libertario, queríamos que siempre los grupos fueran de siempre cinco, y ojalá que todos los grupos estuvieran todos juntos haciendo un círculo grande porque en el fondo ahí hay aprendizaje, donde entre todos sacan una conclusión. (Joven varón secundario, Liceo de Santiago Centro) 
Comprender la participación juvenil estudiantil desde una intencionalidad transformadora revitaliza la agencia de dichos sujetos respecto a la construcción de la comunidad escolar y, junto con ello, permite deconstruir aquellos discursos naturalizados que no observan posibilidad de transformación en los establecimientos educacionales, es decir, «cuando se pierde la conciencia de que las instituciones existentes han sido creadas por la acción humana resultan inimaginables las alternativas y ni siquiera se piensa en posibilidades de cabios» (Wagner, 1997: 143). Por tanto, esto lleva a romper con aquellos discursos que señalan que los agentes educativos estarían condenados a una reproducción acrítica sobre la realidad educativa (Giroux, 1999).

De esta forma, considerar el componente político que subyace en la participación posibilita reconocer los aportes y potencialidades que las y los sujetos juveniles pueden realizar en diversos ámbitos del dispositivo educativo. Ahora bien, cabe señalar que tampoco se trata de esencializar la participación de las juventudes como si fueran sujetos intrínsecamente revolucionarios/as o contestatarios/as, o como quienes fueran los/as encargados/as de transformar aquellos elementos indeseados en la realidad educativa, pues tal como hemos enfatizado, una participación auténtica y sustantiva requiere la activación e involucramiento de todos los actores de la comunidad escolar. Por tanto, avanzar hacia una comprensión en dichos términos requiere ser considerada como un acto cotidiano, reconociendo las multiplicidades de expresiones que adopta y la oportunidad que brinda para la transformación de conflictividades, injusticias o problemáticas que no solo afectan al sistema educativo, sino que también en nuestra sociedad.

\section{REFLEXIONES FINALES}

Iniciamos este trabajo problematizando la comprensión de participación, planteando que se constituye como una noción polisémica y polémica, pues existen diversas aproximaciones que disputan su entendimiento. Ello requiere considerar y clarificar el lugar de enunciación (desde dónde se comprende), el modo en que se ejerce (cómo) y el horizonte que persigue (para qué). En consideración a esto, hemos intentado aportar a dicha discusión desde un acercamiento empírico, posibilitando no solo su discusión conceptual, sino que también observar su operacionalización en las comunidades educativas.

Así, a lo largo del texto, hemos insistido que hablar de participación juvenil estudiantil, lejos de ser un tema que emerge solo en un momento determinado, más bien se constituye como un proceso desplegado de manera múltiple y persistente dentro del espacio escolar. Comprenderla de este modo permite cautelar aquellas reducciones con las que suele operar en la realidad educativa, abriendo posibilidades de avanzar hacia una participación auténtica y sustantiva de las juventudes escolarizadas. 
En ese sentido, si bien hemos centrado la participación juvenil como foco de interés, se ha enfatizado que su comprensión remite necesariamente considerar el carácter relacional y contextual que conlleva. De este modo, se evitan aquellas lecturas adultocéntricas que ponen en relieve el desinterés o baja participación de las juventudes, sin considerar las condicionantes y las relaciones de poder que confluyen dentro de su ejercicio. Así, se trata de incentivar que la comunidad educativa asuma en conjunto el fenómeno de la participación, cautelando lecturas que responsabilizan individualmente a sujetos que no participan.

En consideración a ello, es importante transparentar que hablar de participación juvenil estudiantil resulta una apuesta conceptual y estratégica. Como bien sabemos, el lenguaje construye realidades, por tal razón, anteponer la condición juvenil por sobre la de estudiante supone considerar la participación desde una perspectiva amplia e integradora de diversidad, es decir, contemplar las múltiples racionalidades y sentidos que se anudan dentro de la experiencia escolar. Sin embargo, ello pone como desafío repensar las categorías generacionales con las que se comprenden a los distintos sujetos dentro del espacio educativo, no solo considerando las maneras de ser joven, sino que también las formas en que se construye el sujeto adulto dentro del sistema educativo.

De acuerdo con esto, proponemos que la perspectiva generacional (Duarte, 2019) puede entregar valiosos aportes para avanzar hacia dinámicas de participación genuina, toda vez que ella permite complejizar las interacciones que se despliegan más allá del rol que se cumple en la estructura educativa, reconociendo el modo de relación adultocéntrico que suele caracterizar los vínculos entre generaciones adultas y jóvenes. Este lente para leer lo social complementario a otras perspectivas como género, raza y clase- posibilitaría proyectar algunos criterios de igualdad generacional, potenciando así una participación auténtica y sustantiva. De esta forma, entre las estrategias de relación que considerar se encontraría la aceptación generacional, cooperación generacional y los diálogos intergeneracionales como claves que permitirían avanzar hacia modos de relación en que se valore la diversidad y se promueva el respeto por la equivalencia social de las experiencias y roles.

Esto último conlleva a tensionar los modos de relacionarse entre distintos actores $\mathrm{y}$, por ende, la forma en la que se toman decisiones, cuestiones fundamentales dentro del quehacer pedagógico, así como también dentro de la práctica estudiantil. Así, lo dicho hasta aquí podría dar paso a una reflexión más profunda sobre las posibilidades de repensar la institución escolar, para hacer de ese espacio un lugar más democrático, donde los actores se encuentren, conversen, discutan y también decidan en conjunto.

RECIBIDO: 18 DE DICIEMBRE DE 2019

ACEPTADO: 3 DE ENERO DE 2020 


\section{BibLIOGRAFÍA}

ANDERSON, G. (2001). «Hacia una participación auténtica: deconstrucción de los discursos de las reformas participativas en educación». En Nuevas tendencias en políticas educativas: estado, mercado y escuela (pp. 145200). Ediciones Juan Granica.

ANDRÉU, J. (2001). Las técnicas de análisis de contenido: una revisión actualizada.

BAÑEZ, T. (1999). «Participación ciudadana, sociedad civil y juventud». Acciones e Investigaciones Sociales, 9: 101-124.

COMISIÓN FORMACIÓN CIUDADANA. (2005). Informe final comisión de Formación Ciudadana. Santiago: Serie Bicentenario.

Corominas, J. (1954). Diccionario crítico etimológico. Tomo II. Madrid: Gredos.

DuARTE, C. (2001). «¿Juventud o juventudes? Acerca de cómo mirar y remirar a las juventudes de nuestro continente». Última Década, 8, 13: 59-77. . (2002). «Mundos jóvenes, mundos adultos: lo generacional y la reconstrucción de los puentes rotos en el Liceo». Última Década, 16: 95-113.

. (2019). «Trastrocaciones adultocéntricas y criterios políticos para la igualdad generacional». En K. DUARTE, N. HERNÁNDEZ y Y.

PALENZUELA, Juventudes en Chile: miradas de jóvenes que investigan II (pp. 93-116). Santiago: Social-Ediciones.

DuARte, K. y Álvarez, C. (2016). Juventudes en Chile: mirada de jóvenes que investigan. Santiago: Facultad de Ciencias Sociales Universidad de Chile.

Dubet, F. y Martuccelli, D. (1998). En la escuela. Sociología de la experiencia escolar. Madrid: Losada.

DusSEL, I. (2005). «¿Se renueva el orden disciplinario escolar? Una lectura de los reglamentos de convivencia en la Argentina de la post-crisis». Revista Mexicana de Investigación Educativa, 10(27): 1109-1121.

ESCOBAR, S. (2019). «Participación juvenil: descripciones e interrogantes desde los resultados de la IX Encuesta Nacional de Juventud». Revista INJUV, 7-13.

Franco, P., Franco, B. y GuiLló, C. (2007). «De la participación como elemento de intervención social, a la intervención social como instrumento para garantizar la ciudadanía activa». Monografía, 145: 115-131.

FreIRE, P. (1993). Política y educación. Madrid: Siglo XXI. 
GIROUX, H. (1999). Teoría y resistencia en educación: una pedagogía para la oposición. Madrid: Siglo XXI.

HART, R. (1993). La participación de los niños: de la participación simbólica a la participación auténtica. Unicef.

HERNÁNDEZ, N. (2019). «Lo político en disputa. Una mirada desde las juventudes». En K. DUARTE, N. HeRnÁNDEZ y Y. PALENZUELA, Juventudes en Chile: miradas de jóvenes que investigan II (pp. 93-116). Santiago: Social-Ediciones.

HorkHeIMER, M. (2002). Crítica de la razón instrumental. Madrid: Trotta.

INJUV. (2014). $4^{a}$ Encuesta Nacional de Juventud. La integración social de los jóvenes en Chile 1994-2003. Individualización y estilos de vida de los jóvenes en la sociedad del riesgo. Santiago de Chile.

. (2017). $8^{\text {va }}$ Encuesta Nacional de Juventud. Santiago de Chile.

JARA, C. (2011). «Sobre el falso concepto de participación en educación».

CISMA, Revista del Centro Telúrico de Investigaciones Teóricas, 1: 110.

KrausKopf, D. (2003). Participación social y desarrollo en la adolescencia. San José: Fondo Población de las Naciones Unidas.

Molina, W. y SANDOVAL, M. (2006). «Cultura escolar y cultura juvenil: la (re)construcción simbólica del espacio escolar en la mutación cultural». Revista Temas Sociológicos, 11: 101-123.

NúCLEO INVESTIGACIÓN Y ACCIÓN EN JUVENTUDES. (2016). Consejos Escolares en el contexto de la implementación de la Ley de Inclusión: Posibilidades de Participación para las Comunidades Educativas. Santiago de Chile.

PAlenZuela, Y. (2016). «Imaginarios sociales de jóvenes universitarios sobre su participación ciudadana en redes sociales virtuales». En K. DUARTE y C. ÁlvareZ, Juventudes en Chile: miradas de jóvenes que investigan (pp. 179-195). Santiago: Social-Ediciones.

PALMA, D. (1998). La participación y la construcción de ciudadanía. Santiago: Universidad ARCIS, Centro de Investigaciones Sociales.

Pezo, H. (2019). «¿Liceo disciplinario?: condicionantes para la participación juvenil estudiantil. Una aproximación desde la perspectiva generacional». En K. DUARTE, N. HeRnÁNDEZ y Y. PALENZUELA, Juventudes en Chile: miradas de jóvenes que investigan II (pp. 286312). Santiago: Social-Ediciones.

TENTI, E. (2000). «Culturas juveniles y cultura escolar». Documento presentado en el seminario «Escola Jovem: un novo olhar sobre o ensino médio». Organizado por el Ministerio da Educaçao. Secretaria de Educaçao 
Média e Tecnológica. Coordenaçao-Geral de Ensino Médio. Brasilia, 79 de junio.

WAgner, P. (1997). Sociología de la modernidad. Capítulos 1 y 2. Libertad y disciplina. Barcelona: Herder.

ZARZURI, R. (2016). «Las transformaciones en la participación política de los jóvenes en el Chile actual». En M. A. GARRETóN, La gran ruptura (pp. 133-159). Santiago: Lom Ediciones. 